\title{
Particle dynamics in corrugated rectangular billiard
}

\author{
T. Akhmadjanov, E. Rakhimov and D. Otajanov \\ National University of Uzbekistan, Faculty of Physics, \\ Department of Optics and laser physics, Tashkent, Uzbekistan \\ t_akhmadjanov@rambler.ru
}

PACS 05.20, 05.45

DOI 10.17586/2220-8054-2015-6-2-262-267

\begin{abstract}
The particle dynamics in one side corrugated rectangular billiard system is investigated with the help of numerical analysis. The dependence of chaotic behavior in particle dynamics on the corrugation height $h$ is shown. The focusing mechanism of the corrugated billiard is investigated by analyzing the dependence of the total path on particle incident angle.
\end{abstract}

Keywords: optical fiber, chaos, ray dynamics, corrugated waveguide, Fermi acceleration, billiards.

Received: 2 February 2015

\section{Introduction}

Particle dynamics in waveguides has attracted much attention during past few decades. Such interest is caused by the potential application of waveguides in different topics of contemporary physics, such as optics, cold atom physics and different systems of nanoscale physics. In most of the cases, particle and wave dynamics in waveguides can be modeled by billiards. The latter are the domains confined by hard or soft walls. Dynamics of waves and particles in such a domain strongly depend on the geometry of its walls. In particular, classical equations of motion can be integrable or non-integrable, depending on the geometrical shape of the billiard boundary. Earlier, billiards were the topic of extensive study in the context of nonlinear dynamics and quantum chaos theory [1-5]. It was found that, depending on the shape of the billiard walls, particle dynamics can be chaotic, regular or mixed. A pioneering study of classical particle dynamics in billiards dates back to Birkhoff, who presented a strict formulation for classical mechanics in confined domains [1]. Later, more strict mathematical analysis of Hamiltonian systems in billiards was provided be Sinai [2]. Currently, billiards has become one of the main paradigms in the theory of deterministic chaos. One of the most convenient realistic systems where billiards can be experimentally realized is microwave resonators [4].

Recently, billiards were successfully applied to describe charge transport in nanoscale quantum dots [6]. Besides static billiards, so-called time-dependent billiards has become subject for extensive research during the past decade [7-10]. Such billiards can be realized in atom optics and provide powerful testing ground for classical and Fermi acceleration [11].

Comprehensive and systematic studies of time-dependent billiards were done by several authors in the Refs. [11-15]. Unlimited growth of the particle velocity was not found to be possible for regular (integrable) billiards, while for some types of chaotic billiards, one can observe such an acceleration [16]. The answer for the question whether the energy of the particle can grow to infinity is far from trivial. It depends on the perturbation type on the boundary and the boundary geometry. It was shown by Loskutov, Ryabov and Akinshin in [17] that Fermi acceleration (FA) will be observed in time-dependent billiards if these billiards possess chaotic properties in the autonomous case. Later, this assumption was confirmed for stadium [18] and annular [15] billiards by the same authors. An elliptical billiard was shown to have regular 
dynamics in the case when the billiard has static boundaries, exhibits FA in case when the billiard has time-dependence boundaries. More strict analysis of particle dynamics in timedependent billiards was provided recently by Robnik and co-authors [16], who derived criteria for the shape and time-dependence of the walls from the viewpoint of Fermi acceleration.

Besides Fermi acceleration, time-dependent billiards have generated a certain interest in fiber optics and waveguides. An important and interesting characteristic of the radiation pulses propagating in the optical fiber is the propagation time of rays through the waveguide. Ray dynamics in the corrugated waveguides are becoming more interesting because of the nature of media where the wave can be propagated are more curved by the external fluctuations. This properties help such systems to create some kind of channel for the waves propagating in it, where the signals can be transmitted effectively with minimal loss of information. A similar problem was also considered and the different fractal properties of ray propagation were shown using an underwater acoustic model. They have shown the mechanism of signal cooling when the width of spatial spectra dispersion is significantly reduced. The open stadium billiard model was investigated in [10] using phase space methods. There, they derived the expression for the long time survival probability function for the stadium billiard with a hole [10]. One of example of time-dependent focusing billiards - a stadium-like billiard with periodically perturbed boundary was studied, explained the origin of the increase and decrease of the particle velocity [11].

In this paper, we study particle dynamics in rectangular billiard with a corrugated wall. The phase space portraits for the two dimensional billiard system are obtained for both flat and corrugated billiards. In the next section, we will present the analytical and numerical analyses in detail. The dependence of the particle trajectory and path on the initial incident angle will also be analyzed.

\section{Corrugated rectangular billiard}

The system we want to study is the rectangular billiard (side lengths are $a$ and $b$ ) with a corrugated wall presented in Fig. 1. The main characteristic to be studied is the coordinate of the particle after $N$ collisions with the billiard walls. We analyze phase space trajectory corresponding to such coordinates.

(a)

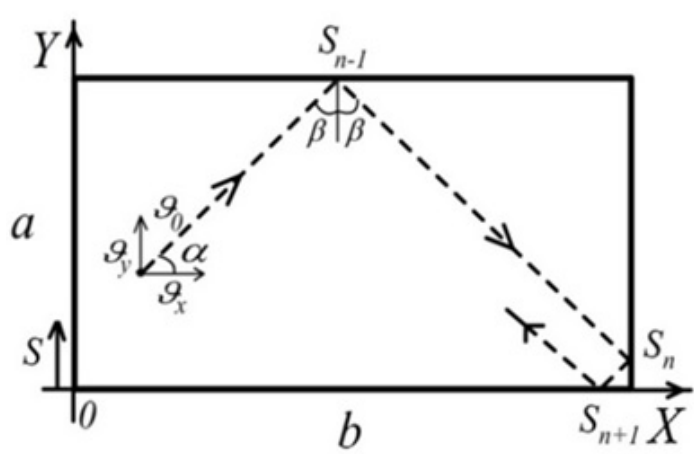

(b)

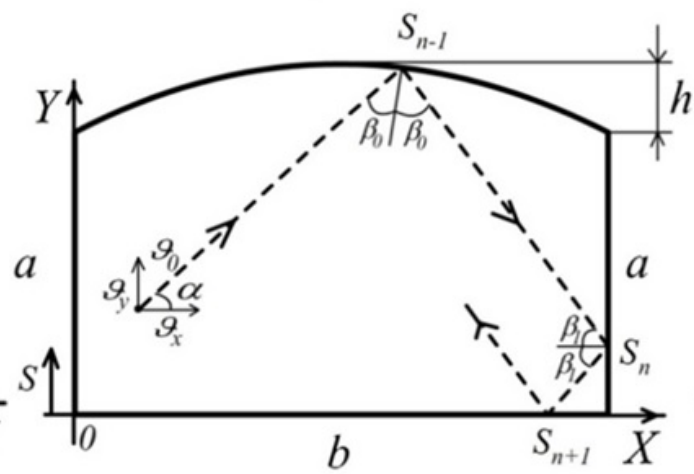

FIG. 1. Billiard model in the form of rectangle with the sides $a$ and $b$ (a) and the same model with corrugated at one side (b)

First, we consider the simple case, a rectangular billiard without corrugation i.e. flat at sides (Fig. 1a), with the sides sizes $a$ and $b$. 
In this case, the boundaries of the billiard will be expressed in $X O Y$ plane as follows:

$$
\begin{aligned}
& y(x)=\left\{\begin{array}{l}
a, \\
0,
\end{array} \quad \text { if } \quad 0 \leq x \leq b ;\right. \\
& x(y)=\left\{\begin{array}{l}
b, \\
0,
\end{array} \text { if } \quad 0 \leq y \leq a .\right.
\end{aligned}
$$

Here $a$ and $b$ are lengths of the billiard sides. ity $v_{0}$ :

Let the particle starts its motion at the point of $\left(x_{0}, y_{0}\right) \in X O Y$ with the initial veloc-

$$
\begin{aligned}
& v_{x}=v_{0} \cdot \cos \alpha, \\
& v_{y}=v_{0} \cdot \sin \alpha,
\end{aligned}
$$

where $\alpha$ is the angle between $v_{0}$ and $v_{x}$ vectors.

Then, one can define the coordinate of the particle after $n$ collision as follows:

$$
\begin{gathered}
\tau_{1}= \begin{cases}\frac{\left(a-y_{n}\right)}{v_{y}}, & \text { if } \quad v_{y} \geq 0 ; \\
\frac{y_{n}}{v_{y}}, & \text { if } \quad v_{y}<0 ;\end{cases} \\
\tau_{2}= \begin{cases}\frac{\left(b-x_{n}\right)}{v_{x}}, & \text { if } \quad v_{x} \geq 0 ; \\
\frac{x_{n}}{v_{x}}, & \text { if } \quad v_{x}<0 ;\end{cases} \\
t_{n}=\min \left(\tau_{1}, \tau_{2}\right), \\
x_{n+1}=x_{n}+v_{x} \cdot t_{n}, \\
y_{n+1}=y_{n}+v_{y} \cdot t_{n} .
\end{gathered}
$$

Phase space portraits are given on the plane of the momentum of particle in the direction of billiard walls, $p=v_{0} \cdot \sin \beta$ and the path of particle, $S$.

Using this map (2), phase-space trajectories can be plotted as in Fig. 2 and 3.

Now, we proceed to the case of corrugated billiard presented in Fig. 1b. The corrugation function is given as follows:

$$
f(\xi)=4 \cdot h \cdot \xi \cdot(1-\xi),
$$

where $\xi=x / b, a, b$ are the rectangular billiard sides and $h$ is the corrugation height.

The boundaries of such a billiard system can be written:

$$
\begin{aligned}
& y(x)=\left\{\begin{array}{l}
a+f(\xi), \\
0,
\end{array} \quad \text { if } \quad 0 \leq x \leq b ;\right. \\
& x(y)=\left\{\begin{array}{l}
b, \\
0,
\end{array} \text { if } \quad 0 \leq y \leq a+f(\xi) .\right.
\end{aligned}
$$

Trajectories of the particle in such billiard can be given in terms of the following map: 
(a)

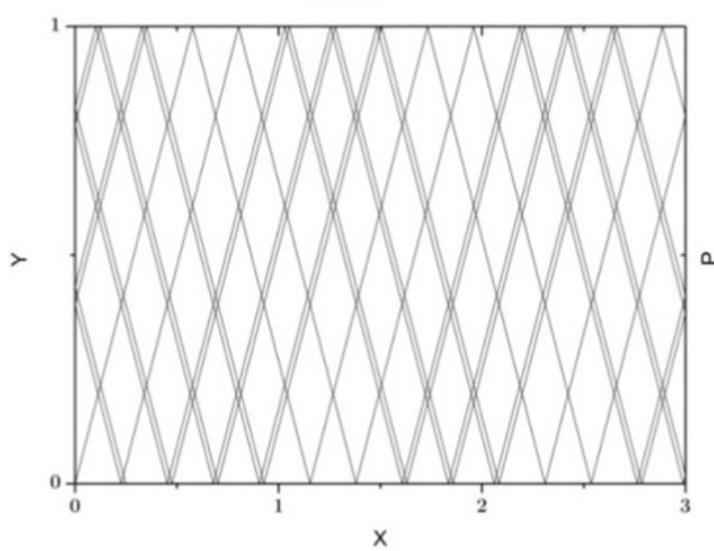

(b)

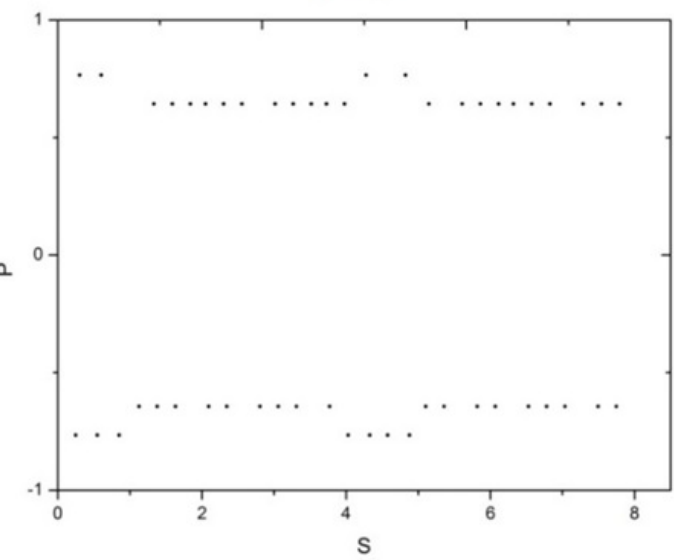

FIG. 2. Trajectory (a) and phase portrait (b) of a particle motion in rectangular billiard for the following billiard parameter values: $a=1 ; b=3$; and initial values for the particle: $x_{0}=0 ; y_{0}=0 ; \alpha=60^{\circ}$

(a)

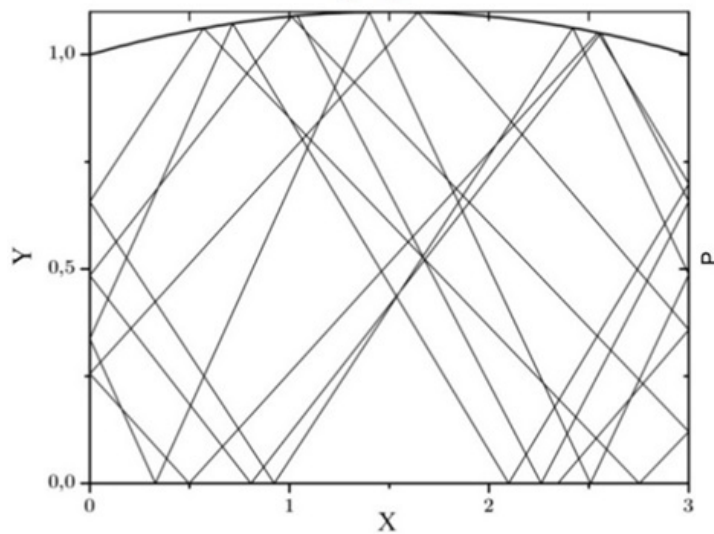

(b)

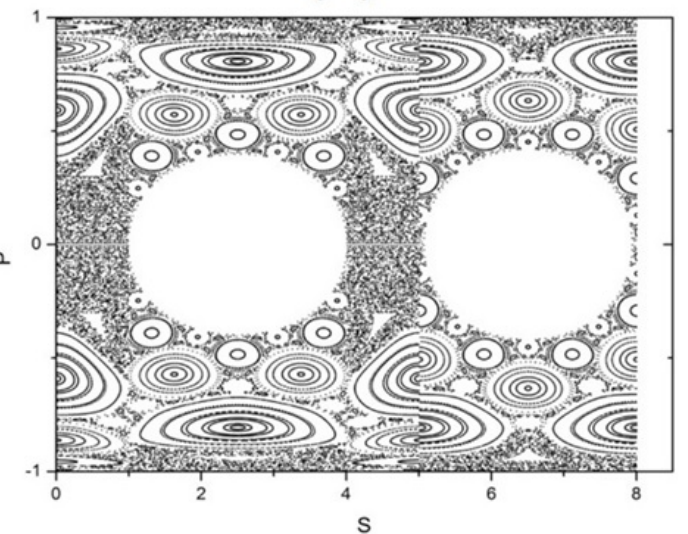

FIG. 3. Particle trajectory (a) and its corresponding phase space portrait (b) for the corrugated rectangular billiard parameters: $a=1 ; b=3 ; h=0.1$

$$
\begin{gathered}
x_{n+1}=x_{n}+v_{x} \cdot t_{n}, \\
y_{n+1}=y_{n}+v_{y} \cdot t_{n}, \\
t_{n}=\frac{1}{2 A}\left(-B+\sqrt{B^{2}-4 A C}\right) .
\end{gathered}
$$

Where the values for the parameters are given as:

$$
\begin{gathered}
A=v_{x}^{2}, \\
B=\frac{v_{y} b^{2}}{4 h}+v_{x}\left(2 x_{n}-b\right), \\
C=x_{n}\left(x_{n}-b\right)+\frac{y_{n}-a}{4 h} b^{2} .
\end{gathered}
$$

Using this map given in (2), also taking into account the expression (5), we obtain the particle trajectory (Fig. 3a) and its corresponding phase space portrait (Fig. 3b). 
One can observe from the Fig. 3 that particle motion is chaotic in this regime, and 'more chaos' can be seen by increasing of $h$.

If we consider the path of particles after $N$ collision, with the initial values of particle coordinate as $x_{0}=0, y_{0}=0$, and for the incident angles range, $0<\alpha<\pi / 2$, we obtain an interesting result for the particle total path, depending on the particle incident angles (Fig. 4).
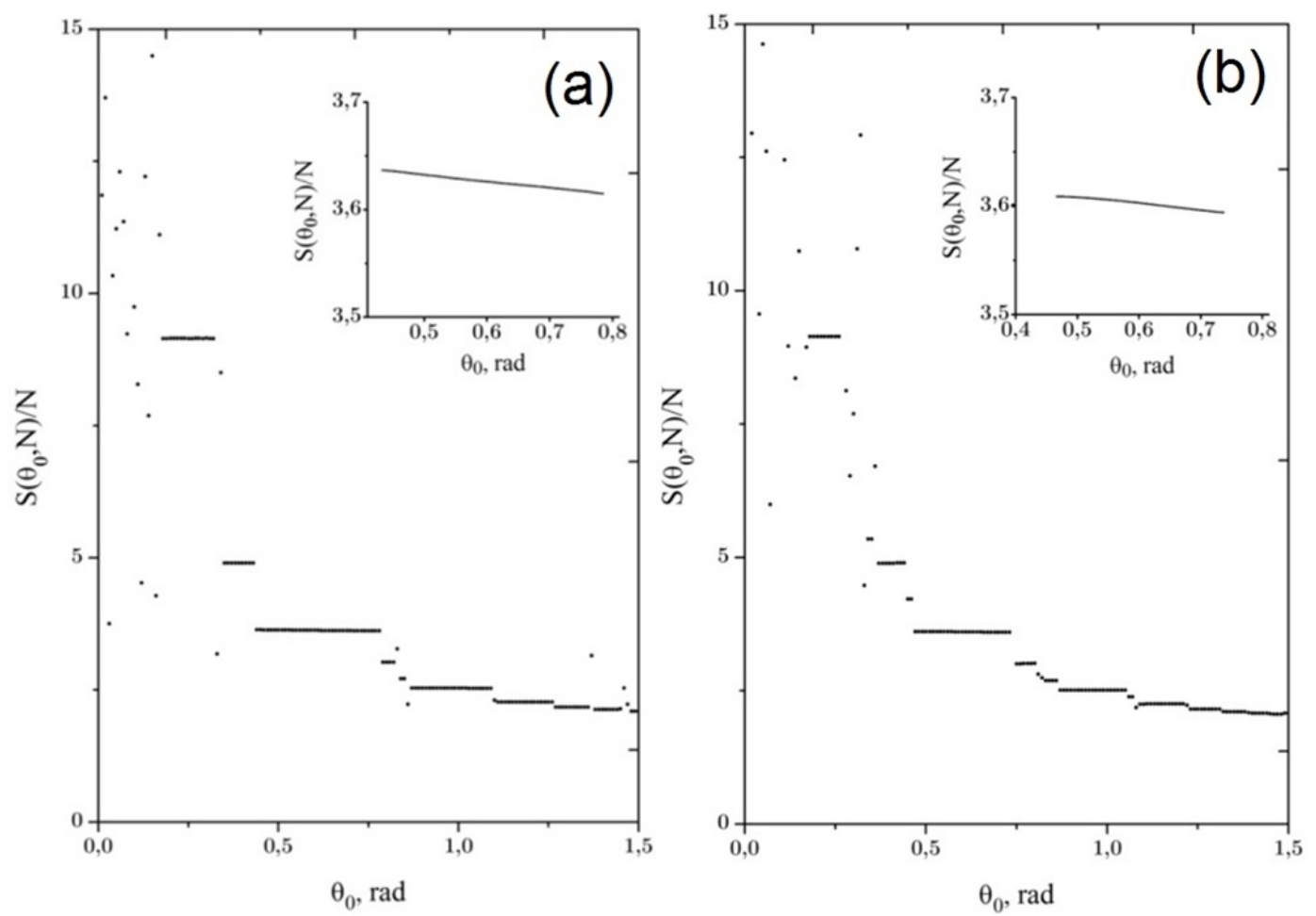

FIG. 4. The dependence of the total path on the incident angle for the parameters $b / a=3$ and $\Delta \theta_{0}=0.01$ of different values of height of corrugation: $h / a=0.03(\mathrm{a}) ; h / a=0.05(\mathrm{~b})$. Scaled region is calculated with the resolution of $\Delta \theta_{0}=0.001$

As can be seen from Fig. 4, 'horizontal lines' in the distributions means that particles will have equal paths after $N$ collisions for different particle incident angles. This gives us the potential to focus the particles in needed directions, thus minimizing signal modes in an optical fiber. Moreover, this allows us to say that chaotic behavior will be increased, according to the increase of the corrugation height relative to the billiard side, $h / a$.

\section{Conclusions}

Thus, we derived two dimensional map presented by Eq. (5) for the rectangular billiard system with a corrugated wall. Using the numerical calculations, we showed the possibility of focusing particle transport by tuning the corrugation paramaters.

The obtained results can be helpful for the problem of transport and chaos control in different waveguides and acoustic systems. 


\section{References}

[1] Birkhoff G.D. Dynamical Systems. Amer. Math. Soc. Colloquium Publ. 9. Providence: American Mathematical Society, 1927.

[2] Sinai Y.G. Dynamical Systems with Elastic Reflections. Ergodic Properties of Dispersing Billiards. Russ. Math. Surveys, 25, P. 137 (1970).

[3] Abdullaev S.S. and Zaslavskii G.M. Classical nonlinear dynamics and chaos of rays in problems of wave propagation in inhomogeneous media. Sov. Phys. Usp., 34 (8), P. 645 (1991).

[4] G.M. Zaslavsky and S. S. Abdullaev. Chaotic transmission of waves and 'cooling' of signals. CHAOS, 7 (1) (1997).

[5] Stoeckmann H.J. Quantum Chaos - An Introduction. University Press, Cambridge, UK, 1999, 384 p.

[6] Nakamura K. and Harayama T. Quantum chaos and quantum dots. Oxford University Press, Oxford, UK. 2004, 216 p.

[7] Loskutov A.Yu., Ryabov A.B. and Akinshin L.G. Properties of some chaotic billiards with time-dependent boundaries. J. Phys. A., 33, P. 7973 (2000).

[8] Loskutov A.Yu. and Ryabov A.B. Particle dynamics in time-dependent stadium-like billiards. J. Stat. Phys., 108 (5-6), P. 995 (2002).

[9] Loskutov A.Yu. Dynamical chaos: systems of classical mechanics. Phys. Usp., 50 (9), P. 939 (2007).

[10] Ryabov A.B. and Loskutov A.Yu. Time-dependent focusing billiards and macroscopic realization of Maxwell's Demon. J. Phys. A., 43, P. 125104 (2010).

[11] Loskutov A.Yu., Ryabov A. and Leonel E.D. Separation of particles in time-dependent focusing billiards. Physica A, 389 (23), P. 5408 (2010).

[12] Loskutov A.Yu., et al. Billiards with time-dependent boundaries and some their properties. Nonlinear dynamics, 6 (3), P. 573 (2010).

[13] Livorati A.L.P., Loskutov A.Yu. and Leonel E.D. A peculiar Maxwell's Demon observed in a time-dependent stadium-like billiard. J. Phys. A, 391 (20), P. 4756 (2012).

[14] Carvalho R.E., de Souza F.C., and Leonel E.D. Fermi acceleration on the annular billiard: a simplified version. J. Phys. A, 39 (14), P. 3561 (2006).

[15] Batistic B. and Robnik M. Fermi acceleration in time-dependent billiards: theory of the velocity diffusion in conformally breathing fully chaotic billiards. J. Phys. A, 44, P. 365101 (2011).

[16] Oliveira D.F.M. and Robnik M. Scaling invariance in a time dependent elliptical billiard. Int. J. of Bifurcation and Chaos, 22, P. 1250207 (2012).

[17] Oliveira D.F.M. and Pöschel T. Competition between unlimited and limited energy growth in a twodimensional time-dependent billiard. Phys. Lett. A, 337, P. 2052 (2013).

[18] Batistić B. Fermi acceleration in chaotic shape-preserving billiards. Phys. Rev. E, 89, P. 022912 (2014). 